\title{
Estudiantes y violencia de sexo/género \\ en la universidad. Propuestas de acción comunitaria \\ en la UNAM (2017-2019)
}

\author{
Students and sex / gender violence in the university. \\ Community action proposals at UNAM (2017-2019)
}

\author{
MAUricio Zabalgoitia Herrera \\ IISUE-UNAM \\ ORCID: https://orcid.org/0000-0003-0806-0887
}

Recibido: 7/8/2019

Aceptado: $15 / 10 / 2019$

doi: https://doi.org/10.20318/femeris.2020.5158

Resumen. Este trabajo surge de la experiencia en dos talleres sobre género y acción comunitaria celebrados en la Licenciatura en Pedagogía de la FFyL de la UNAM. Parte del rol formador y transformador que conlleva la lectura de textos esenciales del feminismo, los estudios de género, las masculinidades y la teoría queer en el espacio del taller. Continúa con una teorización acerca de la constante de violencia como violencia de sexo/género en la educación y la universidad. En un tercer apartado expone los preceptos de las denominadas acciones comunitarias, y su relación con la reconstrucción del tejido social y los regímenes de sexo/género, cerrando con los ejemplos de algunas de las acciones llevadas a cabo por alumnas/os entre el 2017 y 2019, basadas en diversos problemas de violencia en la comunidad universitaria.

Palabras clave: taller crítico, sexo/género, acción comunitaria, violencia, estudiantes.

Abstract. This work is based on the experience in two workshops on gender and community action held in the Degree in Pedagogy of the FFyL of the UNAM. It begins with the formative and transformative role that involves the reading of essential texts of feminism, gender studies, masculinities and queer theory in the workshop space. It continues with a theorization about the "constant of violence" as sex/gender violence in education and the university. In a third section, it sets out the precepts of the so-called community actions, and their relationship with the reconstruction of the social fabric and the sex/gender regimes, closing with examples of some of the actions carried out by students between 2017 and 2019, based on various problems of violence in the university community..

Keywords: Critical Workshop, Sex/Gender, Community Action, Violence, Students

*mauricio.zabalgoitia@gmail.com 


\section{Presentación}

Este trabajo tiene como punto de partida la experiencia en dos talleres sobre género y acción comunitaria, ambos dentro del programa de optativas del Colegio de Pedagogía de la Facultad de Filosofía y Letras (FFyL) de la Universidad Nacional Autónoma de México (UNAM). Su objetivo principal es mostrar, a partir del rol formador y transformador que posee el contacto con la literatura de género, la función social y transformadora que pueden cumplir instancias de educación no formal, como las denominadas acciones comunitarias, con enfoques claros y "duros" de género. A este respecto, los talleres referidos nacieron bajo una inquietud, a la vez teórica, de investigación y didáctica, en cuanto a cómo conciliar aprendizaje e investigación con perspectivas fuertes de género en un ámbito formal de educación -el del programa de la Licenciatura en Pedagogía de la FFyL de la UNAM-, pero con miras hacia la educación no formal y especial atención a una diversidad de problemáticas ligadas al orden de sexo/género reinante. Estas constituyen una diversidad de formas de violencia en espacios, prácticas y relaciones de la vida universitaria. Asimismo, superan los lindes de lo institucional e inciden en un complejo amplio de la experiencia de las/los estudiantes: trayectos en y hacia la universidad, espacios comunes o las aulas, pasando por todas las instancias que conforman el acto de estudiar y ser estudiante en la UNAM.

De acuerdo con esto, en los talleres mencionados ${ }^{1}$ la premisa de partida consistió en leer y debatir textos fundacionales y representativos del feminismo, los estudios de género, las masculinidades y la teoría queer, buscándose en las/los alumnas/os reflexiones hondas y dinámicas que permitieran plantear, desde lo teórico y su discusión o debate, temas, fenómenos y problemas actuales y cercanos -sobre todo en la comunidad universitaria- ligados al orden de sexo/género. Paralelamente, se acometieron lecturas acerca de la noción de educación no formal, y de la variante conocida como "acción comunitaria". Desde estas, se realizaron actividades de observación de las dinámicas cotidianas en la comunidad universitaria y se plantearon proyectos bajo perspectivas y enfoques "duros" de género ${ }^{2}$. Finalmente, bajo la forma de páginas y aplicaciones digitales, campañas gráficas, performances o talleres, sobre todo, se llevaron a cabo una serie de intervenciones e iniciativas de considerable impacto en la FFyL y la Ciudad Universitaria (CU).

Ahora bien, tras el proceso descrito, entre los diversos temas y nociones de género, que tras leer, contextualizar y debatir se posicionaron dentro de los intereses de las/los miembros del taller, podemos adelantar nociones como "techo de cristal" o "micromachis-

\footnotetext{
${ }^{1}$ Los talleres anuales referidos son los siguientes: “Taller de Educación no Formal 3 y 4. Género, estudios culturales y acción comunitaria” (curso 2017-2018) y "Taller de Orientación Educativa I y II. Género, familia y sociedad: taller de acciones comunitarias" (curso 2018-2019).

${ }^{2}$ Consideramos que un proyecto de investigación o de acción comunitaria posee un enfoque "duro" de género cuando es planteado, en todos los estadios de observación, análisis y planeación, tanto científicos como vivenciales, a partir de los llamados "enfoque de género", pero más aún, desde las estrategias teórico-metodológicas del quehacer académico "con perspectiva de género". Para Gabriela Delgado (2008) hay una diferencia entre los trabajos que tienen enfoques de género o que recurren a dicha categoría, así como aquellos que usan la categoría de género y los que surgen de una clara perspectiva; estos tienen un "compromiso feminista" y una posición política con miras a la trasformación del statu quo. Asimismo, emanan desde la comprensión de la "noción de género" (17).
} 
mos", así como formas de violencia o inequidad de carácter individual, profesional y de la vida cotidiana. Cabe mencionar cómo todos los temas trabajados -además las posteriores acciones comunitarias proyectadas y llevadas a cabo - tienen como factor común a la violencia como constante en todas las esferas, relaciones, prácticas y movimientos. A este respecto, de hecho, en los talleres se realizó una dinámica en la que se pidió pensar, a la luz de trabajos fundacionales del feminismo y del género ya leídos y debatidos, acerca de formas de violencia cotidiana, institucional, psicológica, etc., en la que no estuvieran presentes desigualdades, definiciones o prácticas del orden de sexo/género. Las conclusiones de este ejercicio resultan contundentes: no hay hecho o actividad humana en la que el sexo o el género no estén presentes como instancias de/para/hacia la dominación, el control, el abuso o las relaciones con características violentas. Vista así, la noción "violencia de género" podría resultar incluso redundante.

La estrategia, de este modo, consistió en que los/las participantes del taller se situaran como observadores/as, a la vez desde la pedagogía y perspectivas "duras" de género, y como sujetos de/en la universidad. Este proceso tuvo como base las lecturas de feminismo y género, desde las cuales se acometieron actos a la vez didácticos y de transformación de los puntos de vista, realizándose debates, mesas redondas y diversas dinámicas que aportaron un carácter abiertamente crítico al espacio del taller. Desde esta posición se leyeron e interpretaron los casos de violencia, delictivos y de inseguridad de mayor incidencia en el momento, algunos de ellos situados en lo más terrible de una posible "escala" ${ }^{3}$. Específicamente, fueron de gran notoriedad para los talleres el asesinato de la alumna Lesvy Berlín ${ }^{4}$, feminicidio que causó enorme impacto en la comunidad estudiantil, así como el movimiento espontáneo que en México surgió a partir del secuestro, violación y asesinato de la estudiante Mara Fernanda Castilla, en Puebla, por parte del conductor de una unidad de la aplicación de transporte Cabify. A partir de este hecho terrible, en redes sociales como Facebook o Twitter se creó la etiqueta “\#MiCasaEsTuCasa”, tras la publicación espontánea de una mujer que ofrecía su casa como espacio seguro a mujeres en situación de peligro o vulnerabilidad. Este sencillo acto derivo en un movimiento que a nivel nacional e internacional fomentó redes de mujeres organizadas contra los feminicidios.

Tras la lectura crítica y su discusión se fueron conformando las denominadas acciones comunitarias, cuyo punto de vista específico sobre los sujetos, las identidades y sus

\footnotetext{
${ }^{3}$ En un trabajo de 2015, Mingo y Moreno sugieren una "escala de sexismo" desde la que se establece un continuo entre los modos aparentemente más inocuos de violencia de género y aquellos en las que la vida o integridad de la persona estarían en peligro. Las autoras establecen que es el sexismo, como práctica rutinaria, lo que enlaza las manifestaciones de tal posible escala (141).

${ }^{4}$ El 3 de mayo del 2017, Lesvy Berlín Osorio fue asesinada en la Ciudad Universitaria, campus de la UNAM. Tras manejarse diversas hipótesis, muchas de estas provenientes de una posible intención de no incluir el funesto hecho dentro de la categoría feminicidio, apuntando hacia un inverosímil suicidio, se demostró, gracias al apoyo de grupos de mujeres y defensoras de derechos humanos, que en el esclarecimiento del caso se presentaron una serie de irregularidades. Hoy en día un juez ha determinado que el novio de la asesinada fuera acusado por delito de feminicidio agravado. Cabe mencionar que este caso puso en evidencia la falta de protocolos para este tipo de delitos al interior de la universidad, así como las estrategias que la justicia y la sociedad ponen en marcha para interpretar estos sucesos como actos en los que la victima tiene la culpa. Por ejemplo, en las primeras notas periodísticas sobre el suceso, y de acuerdo a fuentes policiales, se dijo que Lesvy era drogadicta y mala estudiante -por tanto merecía morir, podemos leer entre líneas-. Gracias a una intensa labor de la madre, la identidad de Lesvy fue positivamente reconstruida. Hoy se sabe que su novio la golpeó y después estranguló con el cable de teléfono de una cabina pública.
} 
problemáticas, así como la forma en la que aborda aspectos clave en su agenda, como la reconstrucción del tejido social, la toma de consciencia o la implicación de los diferentes actores, establecieron un diálogo nutrido con los problemas y enfoques de sexo/género.

Dicho esto, antes de entrar en detalle en la descripción de algunos de los proyectos que desembocaron en acciones comunitarias dinámicas e incidentes dentro del espacio comunitario de la FFyL y CU, a continuación proponemos, en primera instancia, un breve recorrido por algunas de las lecturas clave del feminismo, los estudios de género, las masculinidades y la teoría queer que tuvieron mayor incidencia. En segundo término, la teorización de la violencia como una constante de sexo/género y su presencia específica en el ámbito educativo y universitario. En un tercer apartado se describen el carácter crítico del taller y los preceptos de las acciones comunitarias, para después dar paso a la descripción de algunas de las acciones más destacadas.

\section{Lecturas de género: de la teoría a la acción}

Un acto a la vez didáctico y pedagógico es el que puede acometerse desde una lectura formal, conjunta y crítica de algunos de los textos fundacionales del feminismo, los estudios de género, las masculinidades y la teoría queer, así como de estudios incidentes, precisos y novedosos que marcan las pautas para ir de la teoría a la acción. Un encuentro planteado de esta manera con la teoría resulta en una actividad mayúscula de carácter transformador, pues redefine los contornos de lo que conocemos como realidad, desdibuja las fronteras de los sujetos y las identidades, y pone de manifiesto las férreas construcciones del engranaje humano y de los mecanismos del poder, el dominio, la opresión y, claro está, a la violencia como una constante, como una estrategia de dominación a priori de sexo/género. Los textos "duros" de género comparten con otros de la teoría crítica, de la filosofía o del marxismo la posibilidad de una lectura vital con carácter transformador, ahí en donde toman como objeto los espacios y mecanismos mediante los cuales se definen, condicionan o liberan los lugares esenciales para la vida. En el contexto específico del aula universitaria, leer sobre la condición femenina, el patriarcado, las masculinidades, las identidades, la diferencia sexual, la separación del mundo y sus subjetividades en esferas, el papel institucional y de poder de cuestiones aparentemente tan dispares como la literatura, la ley, los cuerpos o la educación superior, entre otras tantas, no sólo pone en jaque muchos de los saberes aprendidos, sino que, de hecho, provoca que el acto de ser y vivir sea visto desde una nueva, estimulante, y quizá aún no conocida perspectiva. Esta es una cuestión que sin duda destaca en las estudiantes social y culturalmente identificadas como mujeres, pero que resulta especialmente llamativo en los varones. En pocas palabras, el poder transformador de la literatura "dura" de género no funciona igual en "ellas" que en "ellos"; dice lo mismo, pero no significa de la misma manera. Mucho menos en un tiempo en el que las mujeres, y más las universitarias de ciencias humanas o sociales, están más informadas, sensibilizadas, unidas, organizadas e interesadas en el feminismo, en el género y en la transformación de la realidad. Muchas de ellas, de hecho, 
viven ya la experiencia estudiantil desde tal perspectiva, aunque esta no coincida, en realidad, con los temas, saberes y métodos que conforman el currículum de los programas universitarios. El de la Licenciatura en Pedagogía no posee ni una sola asignatura troncal u obligatoria que aborde las teorías, metodologías y saberes del feminismo o cualquier otra expresión del género. Es en algunas optativas que se tocan de manera directa o indirecta temas o enfoques de género.

A lo anterior hay que sumar otro de los aspectos ligados a la lógica de una educación superior "genderizada", siguiendo la marca trazada por Michael Kimmel (2017), que es el que establece a la matrícula de Pedagogía como femenina. Así, en la experiencia específica de los talleres, en el primero había alrededor de 20 alumnas y tres alumnos. Para la segunda o tercera sesión sólo permaneció uno de los varones, el cual se manifestó bajo una identidad gay desde el primer día. Desde esta posición, y su interés y conocimiento del género, aportó valiosos aspectos al trabajo colectivo. En el curso siguiente, de 18 inscritas/ os cuatro fueron varones. Tres permanecieron durante todas las sesiones, manifestando una dispuesta sensibilidad a la maleabilidad de los roles y comportamientos de sexo/género. Ellas, desde una natural diversidad y biografías, o sabían mucho de temas de género y feminismo, o habían leído algunos bestsellers sobre el tema, o visto alguno de los varios documentales que comienzan a producirse y exhibirse en plataformas como Netflix, o incluso habían leído textos teóricos del feminismo y participado en asambleas y grupos organizados bajo esta perspectiva ${ }^{5}$.

Entre los textos "fundacionales" trabajados en el taller podemos destacar algunos, buscando entrecruzar sus conceptos o debates con esa transversalidad de violencia en los acontecimientos observados en la comunidad universitaria, así como en los temas que por actualidad o experiencia atrajeron las miradas investigativas de sexo/género de las/los estudiantes. En primer término, La dialéctica del sexo (1970) de Shulamith Firestone, que funciona como uno de los textos de partida. Siendo un libro escrito por la autora a los 25 años -de ahí, acaso, la efectividad con la que dialoga con las/los estudiantes de licenciatura- en este acomete una revolución dentro del feminismo por estar considerado como una bisagra que reflexiona los temas de base de la primera ola desde la óptica marxista de la segunda. La vigencia, a este respecto, de una de las categorías clave que Firestone marca como fundamento de la opresión hacia las mujeres, la de la maternidad, conserva una denotada actualidad; acaso más en sociedades en las que la desigualdad económica y de educación deriva, como sucede en todos lo casos, en diferencia por cuestiones de sexo y de género. Dicha "maternidad" es concebida desde Firestone como socialmente construida, y atribuida a las mujeres bajo preceptos a la vez políticos y biológicos. Su aportación, sin ir más lejos, orilla la cuestión de la lucha de clases al espacio de la diferencia sexual. Como texto fundacional del feminismo radical plantea las bases para situar a la mujer

\footnotetext{
${ }^{5}$ En términos de la "genderización" de la matrícula, de las asignaturas, de los contenidos, de las/los autoras/es y de las identidades en sí del género -mujer, hombre, gay, lesbiana, trans, queer, fluido, etc.-, una conclusión constante en ambos talleres derivó en un objetivo de la perspectiva de género "aún por instaurarse" en un espacio como el de la FFyL: el de hacer llegar los mensajes más incisivos y transformadores a quien también, y de forma urgente, debe escucharlos; a "ellos", los cuerpos parlantes socioculturalmente percibidos -y autopercibidos - como hombres-varones heterosexuales o cisgénero.
} 
como la clase oprimida. En el contexto universitario estas aportaciones no sólo avivan las intenciones revolucionarias y de transformación en la natural condición estudiantil en las humanidades, y en los últimos semestres de Pedagogía, sino que también invitan a reflexionar en cuanto a logros y retrocesos en términos de las condiciones sexopolíticas que continúan diseminándose en la construcción de las mujeres. La maternidad, de hecho, fue una referencia constante y un tema central en las discusiones, de ahí los cuestionamientos: ¿hasta dónde replantearla o deconstruirla? ¿Cómo armonizarla con los tiempos actuales? ¿Cómo vivir el hecho de querer ser madres o padres? ¿Cómo construir paternidades desde nuevas formas de habitar el mundo? En términos de violencia de sexo/género los debates se plantearon en cuanto a la relación entre maternidad, como actividad a la vez sublimada socialmente y sometida al escrutinio, y dominación, control, producción, reproducción y la lógica de consumo.

Otro de los trabajos incidentes es La mística de la feminidad de Betty Friedan, publicado en 1963 en EUA. En este se establecen las bases históricas fundamentales de la diferencia sexual, de la separación de esferas, y se trazan los principios para comprender el orden de la realidad como resultado de una lógica binarista que organiza no una parte del mundo, sino al mundo como tal. En los talleres, a partir de ese "malestar que no tiene nombre" como condición que aqueja a la mujer a pesar de una ilusión de paridad política y social, deriva en discusiones sobre el trabajo doméstico, y hacia conceptos posteriores como el de la "doble jornada". Esto en las/los alumnas/os provoca la revisión de sus referentes familiares y de sus propias experiencias, pues hay los que ya viven en pareja o bajo otra forma de organización social -como el compartir hogar-. Temas como las tareas de casa, la crianza, el aborto o el trabajo derivan en formas vigentes de violencia sexual y genérica, muchas de las cuales se descubren, incluso, como invisibles, como no problemáticas, o como concesiones que las mujeres continúan haciendo de manera "natural". En términos de masculinidades, Friedan ya apunta al trabajo que debe realizarse con los sujetos socialmente construidos como hombres-varones, pues ya ve, como parte sustancial del problema, temas que a día de hoy están de actualidad: los vínculos de amistad entre ellos, los sentimientos y sus manifestaciones, la reproducción de la violencia en sus relaciones afectivas, etc. Este texto fundacional revela cómo imperan serios desajustes, tanto en la educación formal como en la cotidiana y social, entre la agenda del feminismo de la segunda ola y el de la cuarta o los feminismos actuales. A este respecto, en México estaríamos viviendo una suerte de distintas velocidades de sexo/género, con espacios de franca apertura y otros de mantenimiento de férreas tradiciones en las que la mujer siempre lleva desventaja. También en cuanto a cómo se han perfeccionado mecanismos para mantener el control y la opresión en esos espacios híbridos del acontecer diario. Desde el punto de vista masculino, los debates derivaron en cuanto a reconocer la implicación que el varón perpetúa en un círculo de inequidad que va de la casa a la escuela y al trabajo.

Estos son apenas dos ejemplos de cómo se trabajó la teoría como instancia transformadora, y como guía para observar, con perspectiva de género, los espacios del presente desde el pasado feminista. Esta es una tradición que sin duda debería primar por sobre otras. Aquí es imposible reseñar todos los trabajos abordados, pero sí queremos destacar 
otras fuentes "duras", como la Política Sexual de Kate Millet, publicado en 1969, en donde se va de la literatura al psicoanálisis, pasando por la educación o la antropología, para mostrar la violencia de sexo/género subyacente en todo orden social o cultural. Dicha política sexual, Millett la descompone a partir de sus aspectos ideológicos, biológicos, sociológicos, psicológicos y económicos, resultando ese mundo de tensiones políticas que se reconstruye en extremo familiar para el alumnado que se educa en el México de la segunda década del siglo XXI. En la lectura del segundo capítulo, "Teoría de la política sexual”, las/los asistentes al taller comprobaron con ejemplos cotidianos cómo el sexo es una categoría social impregnada de política; y como el dominio sexual sigue siendo, como Millet sugiere, "la ideología más profundamente arraigada en nuestra cultura" (70).

También hay que destacar la lectura de textos densos, como "Tráfico de mujeres" de Gayle Rubin, pero que promueven esa acción transformadora en las/los estudiantes. Como es sabido, en este capital trabajo se echan abajo las propuestas de Engels sobre la familia, de Freud sobre los mitos de la sexualidad, de Lévi-Strauss sobre las estructuras de parentesco y el tabú del incesto, mostrándose con esto cómo subyace un principio violento de diferencia sexual en todas las culturas conocidas y estudiadas por la antropología. Para la reflexión en el espacio del taller, este trabajo sirve para comprender cómo es que lo masculino siempre queda mejor situado en los juegos de oposición binarios, entre los cuales destaca ese mayúsculo, que la autora nombra, el denominado sistema sexo/género $^{6}$. Esta reveladora aportación hace que toda lectura teórica, crítica o transformadora de la experiencia humana y sus manifestaciones culturales sea leída bajo la lupa del "ojo feminista", incluso las relaciones universitarias.

También está el influyente El género en disputa (1990) de Judith Butler, en donde no sólo el género se presenta como una construcción cultural, sino el sexo en sí se comprende como algo humanamente concebido, pero que se hace pasar por natural para establecer una normativa (de género). Tras esta lectura, en el taller se sientan las bases centrales del feminismo postestructural y queer, llegándose a comprender la "teoría performativa del género". Desde esta se cuestiona la aparente naturalidad del sistema binario sexo/género, y se revela cómo es el que el género es el resultado de acciones que emanan de categorías centrales como hombre o mujer. Con esto, las realidades de la experiencia universitaria son leídas como el resultado de un despliegue performático en el que todos los significados pueden deconstruirse.

Además, se trabajaron en profundidad importantes textos, como el fundacional sobre masculinidades de Raewyn W. Connell (1995), y que referiremos en el próximo apartado en cuanto a la teorización sobre la violencia. El fundacional de la teoría queer, de Eve Kosofsky Sedgwick, "A(queer) y ahora" (1993), trabajo en el que sienta las bases de su noción de esta teoría, mostrando cómo lo afectivo y las narrativas personales constituyen un espacio de discusión epistémica y política muy destacado, sobre todo desde una per-

\footnotetext{
6 "He llamado a esa parte de la vida social el `sistema de sexo/género', por falta de un término más elegante", y "como definición preliminar, un `sistema de sexo/género’ es el conjunto de disposiciones por el que una sociedad transforma la sexualidad biológica en productos de la actividad humana, y en el cual se satisfacen esas necesidades transformadas" (Rubin, 2000: 97).
} 
spectiva crítica de sexo/género. En una línea aún más de vanguardia, textos como el Manifiesto Contrasexual (2002) de Beatriz Preciado -ahora Paul B.- tienen un remarcado éxito, no sólo porque muestran cómo lo queer puede situarse políticamente frente a la teoría que pretende ser liberadora -el marxismo, la deconstrucción-, sino que plantean que en la lucha contra la diferencia sexual el juego es una herramienta de enorme efectividad ${ }^{7}$.

\section{La constante de violencia como violencia de sexo/género: educación y universidad}

Otra de las motivaciones surge de la complicada actualidad nacional y urbana, así como de la comunidad universitaria ligada a la UNAM. México, desde hace por lo menos tres décadas, ha visto incrementarse hasta niveles impensables los índices de violencia, inseguridad y delincuencia, ligados, claro está, a cuestiones de inequidad, aumento de la pobreza, criminalización del estado, etc. Si retomamos la cuestión de la constante de violencia como a priori de sexo/género, los puntos de vista desde los que determinados sucesos son percibidos no sólo cambian considerablemente, sino que el hecho en sí del género se revela como esencial. El caso del feminicidio de Lesvy Berlín es un ejemplo pertinente.

En términos específicos de violencia de género, que es como se ha tendido a acuñar la diversidad de actos que emanan de definiciones, identidades y ejercicios de poder, dominación, abuso, violación o asesinato ligados al ordenamiento de los sexos, sus definiciones y normas, o de sus expresiones y definiciones genéricas, hoy en día existe un determinado consenso en cuanto a su operación en las instituciones de educación superior; y específicamente en la UNAM. A este respecto, el presente trabajo no pretende ser un informe concienzudo o un estudio específico -y cualitativo- de la violencia de género, o de aquella ligada a los ámbitos escolares -más específicamente los de la universidad en México-. Sin embargo, para contextualizar los tiempos que corren, podemos traer a colación cómo Nancy Lombard (2018), en la introducción a The Routledge Handbook of Gender and Violence, y con la idea de definir lo que conocemos como "gender-based violence" -un problema a la vez antiguo y siempre vigente, expresa-, recuerda que el género es constituido a la vez como una estructura social -desde las instituciones-y como un constructo discursivo en el que el lenguaje y las acciones cobran sentido. Ahí los roles, comportamientos, posiciones, responsabilidades y expectativas adscritos a hombres (y niños) y mujeres (y niñas) conforman ideas diferenciadas en cuanto a cómo se tienen que comportar y actuar cada uno de estos pares (Lombard, 2018: 1). Ahora bien, pero el género no sólo es la definición de cada par, sino las relaciones que entre estos se establecen. Para comprender esto para ella es crucial la propuesta que Connell (1987) ya había hecho décadas atrás; la de concebir al género como ligado a regímenes bajo los que dichas relaciones

\footnotetext{
${ }^{7}$ En términos generales, nos preguntamos: ¿cómo es posible que en el siglo XXI, en la UNAM, una/un estudiante de humanidades o sociales lea a Marx, a Freud o a Foucault, pero no lo que el feminismo, los estudios de género, las masculinidades o lo queer tienen que decir o le replican a estos autores? ¿No se está ignorando deliberadamente una tradición que plantearía una educación otra, una pedagogía crítica y a la vez radical sin precedentes? Si como sostenemos, la violencia de sexo/género está en la base de todas las formas de violencia, el papel de la educación superior debería ser formar en los conocimientos que permiten su comprensión, así como las claves para su verdadera deconstrucción.
} 
funcionan: el trabajo, la educación o la familia (2018: 2). En estos, asimismo, se reproduce y perpetúa la posición dominante de los hombres y la subordinación de la mujer (2). A esto se suma la también pionera visión de Kimmel (1987), para quien las adscripciones de género se aprenden y disputan con el paso del tiempo y con los cambios en la cultura (2018: 2). A esto suma la conocida noción de Connell de "masculinidad hegemónica", la cual establece las variantes entre las masculinidades dominantes, y la más dominante, a su vez, dentro de estas. Siguiendo a Connell, "[t]o maintain this priviled position and the current gender order, hegemonic masculinity must embody stereotypical masculine traits of power, dominance strenght and authority" (en Lombard, 2018: 2). Es en este complejo en donde la feminidad y las masculinidades no hegemónicas son concebidas como débiles, y la violencia y agresión son normalizadas y priorizadas como piezas clave en el orden específico de las masculinidades. Con esto lo que se demuestra es que la violencia de género, estudiada desde una perspectiva "dura" de género y no como una forma más de la experiencia humana, es un tema no sólo del feminismo, sino de las masculinidades, pues son los sujetos socioculturalmente concebidos como hombres-varones quienes la suelen ejercer y mantener.

Pero antes de volver al "género de la violencia", como la llama Kimmel (2017), cabe mencionar cómo para Lombard la violencia, en una rápida construcción, es perpetrada en múltiples formas, y es, como adelantamos, uno de los problemas más graves que aquejan a la humanidad. Citando a Kenway y Fitzclarence: "It is increasingly understood that violence occurs along a continuum and involves physical, sexual, verbal and emotional abuses of power at individual, group and social structural levels" (en Lombard, 2018: 2). Aquí proponemos que ese continuo, de hecho, es un régimen ya de sexo/género de por sí, pues si bien la violencia puede ejercerse en términos de raza, etnia, clase social, nacionalidad, etc., el género y su organización cultural y sexual son las invariantes ${ }^{8}$. Ahora bien, más allá de que es en el hogar y en la familia en donde las mujeres sufren la mayor violencia, la escuela y las instituciones educativas destacan dentro de los regímenes de género en donde también son las mujeres quienes están mayormente expuestas a diversas y a veces naturalizadas formas de dominación y abuso, algunas incluso mortales, como bien hemos referido en los casos recientes. En la introducción de Lombard se insiste en cómo la violencia -de género y contra las mujeres- se sigue negando u ocultando en un mundo en el que los números expresan que cada día más mujeres sufren violencia doméstica, violaciones, acoso y explotación sexual. Esto sin olvidar a gays, lesbianas y a todo el haz de masculinidades no hegemómicas o diversas.

Para Kimmel, en su capital aportación del 2000, The Gendered Society, la educación se sitúa en un punto muy destacado dentro de las "gendered institutions". De hecho, la sitúa después que a la familia y antes que a la religión. Para él, es en el salón de clase en

\footnotetext{
${ }^{8}$ Con esto no queremos opacar el hecho de que marcar una violencia como violencia de género lo que busca hacer visibles es cómo quienes normalmente la sufren son las mujeres. No hay que dejar fuera el hecho de que la noción "violencia de género" surge citando la experiencia de mujeres en todos los países y estratos socioculturales y étnicos del mundo. El Consejo Europeo la define como una "manifestation of historically unequal power relations between women and men, which have led to domination over, and discrimination against, women by men and to the prevention of the full advancement of women" (en Lombard, 2018: 2).
} 
donde se marcan, definen, instauran y reproducen las pautas para establecer que hombres y mujeres son categóricamente diferentes. Kimmel se refiere a los modos en los que se educa a los varones y a las mujeres en sistemas aparentemente igualitarios, pero sofisticada y efectivamente desiguales. También en cuanto a cómo las áreas son marcadas por el género, p. ej. las ciencias y las matemáticas como disciplinas para ellos; el español o la historia para ellas, y de acuerdo con formas de violencia epistémica que reditúan en modos de violencia cultural o física; o con los imaginarios de género instaurados en el fondo de la educación que avalan un régimen de género desde el que la constante de la violencia se ampara en ideales y mitologías de masculinidad y feminidad. Esto se perpetúa hasta la universidad, en donde no sólo las matrículas se mantienen "genderizadas" -pedagogía, idiomas, comunicación como espacios de mujeres; ingenierías, derecho, medicina como el quehacer de hombres-, sino como un lugar destacado en donde ellas y las identidades diversas de sexo/género lo siguen teniendo más complicado a la hora de destacar. A esto hay que sumar, como se ha puntualizado desde los talleres, naturalizados modos de acoso, amenazas, y hasta abusos y violaciones en los que casi nunca se castiga a los culpables.

En esta línea, Araceli Mingo (2010) ha ofrecido un panorama preciso en cuanto a la magnitud y las formas que la violencia escolar manifiesta al estar ligada a aspectos de género. Para esta autora, la violencia es un hecho cotidiano en la educación, y tras revisar encuestas centradas en México y otros lugares del mundo, el sexo se manifiesta como una constante bajo la cual se perpetúan diversas conductas violentas; así, "[...] el sólo hecho de ser mujer u hombre incrementa o disminuye el riesgo de recibir o cometer alguna de estas conductas" (26). Mingo se concentra en la naturalización de la violencia, en cómo está se encuentra inmersa en los "usos y costumbres" y por tanto se solapa bajo cierta "ceguera" que afecta a los actores que la ejercen o la padecen. Así también, a que en su reconocimiento o procesamiento participan cuestiones como la negación o el silencio, lo que dificulta comprender a la violencia o lidiar con ella. En este complejo "[h]a de considerarse que la humillación, el susto, el dolor, la rabia, la parálisis, la vergüenza o cualquier otra vivencia que va de la mano de un golpe, de un insulto, de un grito, de un tocamiento forzado, de una mirada que ofende, o de un gesto que disminuye a quien lo recibe, dejan de una u otra forma su registro en el cuerpo [...]" (26). Esto último describe con claridad el tipo de fenómenos cotidianos, institucionalizados o sumidos en una lógica de impunidad que las/los alumnas/os de los talleres identificaron con astucia en las relaciones diarias de la vida universitaria y sus capas formales e informales, lo que se podrá apreciar mejor cuando describamos algunas de las acciones comunitarias ideadas y llevadas a cabo. Gracias al repaso de Mingo, cuestiones estudiadas desde una idea general de la violencia adquieren una significación más densa desde el punto de género; de este modo, desde las "violencias pequeñas o cotidianas" o las "microviolencias" de Debarbieux (en Mingo, 2010: 27), o el llamado bullying, hasta llegar a modos de "violencia simbólica", los números y definiciones vuelven a apuntar a los hombres-varones, pues son ellos quienes ejercen la violencia en prácticamente todos los casos ${ }^{9}$.

\footnotetext{
${ }^{9}$ Cabe decir que según un trabajo de Kimmel (2004) referido por Mingo, en EUA ellos mueren más por homicidio y cometen más suicidios, mientras que ellas acusan elevados niveles de contactos y relaciones sexuales contra su voluntad.
} 
Acercándonos a la universidad, la aportación de Mingo deriva hacia los actos de acoso presentes en los contextos de educación superior, p. ej.: "hacer comentarios sexuales, chistes, gestos o miradas; diseminar rumores sexuales y llamar a otros y otras: gay o lesbiana" (2010: 30). Estas distintas prácticas, que conforman el "hostigamiento sexual", como bien expresa la autora, si bien suelen ser mayormente acometidas por estudiantes también incluyen al personal docente o de otros sectores laborales de la universidad -lo que se corroboró en los talleres-. La cuestión más compleja en esta deriva es la que estudia, siguiendo un trabajo de Billie Wright y Linda Weiner, "los mitos que se utilizan para descargar de responsabilidad a quienes abusan de la situación de poder que guardan respecto a estudiantes, colegas o personal administrativo" (en Mingo, 2010: 30); así también el "silenciamiento cómplice" entre varones o la institución $(30)^{10}$. Aquí, una vez más las reflexiones derivan hacia los hombres-varones y las masculinidades. A través de diversos estudios, centrados en la cultura anglosajona de EUA, se observa cómo los actos de hombría igualmente están insertos en una lógica de violencia; cómo los ritos de la masculinidad buscan mantener los privilegios del grupo sexual dominante; cómo se ejercen los mecanismos para controlar y ser controlados; cómo la exigencia de heterosexualidad es producto del ejercicio de diversas formas de violencia; o cómo los roles de la masculinidad, a su vez, son el producto de ideales: ser el proveedor, mantener distancia emocional con la pareja, ser el guía, marcar a las mujeres como objetos sexuales (40-41) ${ }^{11}$. Destacamos, finalmente, el recorrido que se hace por formas más sutiles de violencia y que Mingo denomina como "indirecta". Siguiendo con su atenta lectura de estudios destacados, repara en nociones como la de "microinequidades" de Mary P. Rowe, siendo en la universidad en donde muchas veces se ensayan y perfeccionan; a decir, el atribuir a un hombre las ideas o aportaciones de una mujer, el pensar sólo en varones para determinadas tareas destacadas, el que las mujeres deban tomar determinados caminos para volver a sus casas, etc. (42). Por último, resaltamos el término "micromachismos", que Mingo trae a colación citando a Luis Bonino (2014). Se trata de comportamientos o acciones de "baja intensidad", muchas veces no identificados, que favorecen el dominio de los hombres sobre las mujeres (2010: 43). Esta noción generó mucho interés en el espacio de los talleres y la preparación de las acciones comunitarias. Sin duda, el haz de posibles formas de violencia solapada, normalizada o diaria que involucran estas microformas resultan especialmente llamativas en un espacio que se presume igualitario, equitativo y sensible, como la FFyL de la máxima casa de estudios en México.

Kimmel, en el referido trabajo del 2000, habla sobre el "género de la violencia". Al referir a las diferentes matanzas acontecidas en EUA en la década de los 90, perpetuadas

\footnotetext{
${ }^{10}$ En la parte centrada en México, Mingo recurre a una diversidad de estudios y encuestas dentro de las cuales podemos destacar los altos índices de violencia de género intrafamiliar, pero también en relaciones que afectan a la juventud en los ámbitos formales e informales de la educación, p. ej.: violencia en el noviazgo, violencia psicológica, violencia e insultos por estereotipos o identidades de género, hostigamiento y agresiones físicas (34).

${ }^{11}$ Destacan, a su vez, determinadas estrategias para hacer ver que "a ellos nadie los controla", como pueden ser "bromas, torneos verbales, el uso de lenguaje sexista o realizar sabotaje para mostrar autonomía". Asimismo, "el uso de violencia física y verbal con mujeres que no muestran la sumisión esperada por sus parejas"; o "la misoginia que se observa en algunos grupos gay [como] una forma de distanciarse de las mujeres y del lugar subordinado que éstas ocupan en las jerarquías sociales" (41; cursivas del original). Igualmente, las figuras del héroe o el guerrero, estudiadas por autoras del feminismo, son traídas a colación para ligar masculinidad con violencia física y pelea (41).
} 
todas por jóvenes varones, además de actos terroristas, actividades del crimen organizado en México, o a hechos de homofobia en diversos lugares del planeta, reflexiona sobre si en alguno de los noticieros o periódicos en los que se manifiestan actos violentos se menciona que "[...] every single one of these terrorists, suicide bombers or racist gang members is male?". 0 si "Do they investigate how ideologies of masculinity may have contributed to the motivation for such heinous crimes? (449). También muestra cómo estas cuestiones no representan un tema, siendo el hecho de que todos estos sujetos sean varones es tan natural que no necesita ninguna reflexión o análisis ${ }^{12}$. Para Kimmel, la cuestión se resume en que la violencia está ligada al género y el problema es, básicamente, uno de "male violence" (450). En términos específicos de la violencia contra las mujeres, el autor recuerda cómo el hombre-varón aprende que esta es una forma aceptada de comunicación entre hombres, así como entre hombres y mujeres.

En el contexto mexicano debemos mencionar algunos trabajos recientes que han dado cuenta de la relación entre violencia de género y estudiantes. De los autores Francisco Zamudio, Marco A. Andrade, Roxana Arana y Arturo Alvarado, en 2017 se publica un reporte de investigación en el que se propone un "índice" para medir la violencia de género entre estudiantes universitarios/as. Tras un interesante enfoque cuantitativo, los resultados de un cuestionario aplicado a estudiantes de la Universidad Autónoma de Chapingo revelan una preocupante realidad de violencia de sexo/género, mostrándose no sólo consecuencias físicas, sino emocionales y sociales, en las que son las mujeres las peor situadas. En 2012, se presentó un trabajo también multiautorial, proveniente de un proyecto de investigación interuniversitario ${ }^{13}$. La finalidad de este ambicioso proyecto fue el crear un "marco conceptual" que permitiera abordar los problemas ligados a la violencia de género en la universidad desde un frente común, considerándose una visión amplia de la violencia, la cual incluye a la directa, la estructural y la cultural. En este marco, se trabajan cuestiones que parecen reproducirse en todas las IES, como son: piropos ofensivos, tocamientos, violación, insultos, amenazas, golpes; así como formas más sofisticadas de violencia estructural, como la "tipificación sexual por campos del conocimiento" (92), o de lo que la que denominan "cultural", que sería la que explica la inequidad de hombres y mujeres en los altos cargos de las IES. El llamado "techo de cristal", como se ha mencionado, es un tema que llama mucho la atención en las estudiantes de los talleres ${ }^{14}$.

\footnotetext{
${ }^{12}$ Asimismo, a la hora de citar una investigación de 1993 sobre juventud y violencia, enlista una serie de factores como la "frustration, lack of social skills, being labeled as a 'dumb', poverty, abuse, neglect, drugs, alcohol, violent video games, and the availability of guns. Neither of these blue-ribbon panels'reports mentioned the Word 'masculinity'" (449).

${ }^{13}$ Bajo el proyecto: "Clima escolar y violencia de género en las IES de algunos estados del centro-occidente de la República Mexicana: un estudio comparativo”, financiado por el CONACYT, publican: Rosa María González, Catalina Suárez, Marisa Polanco, Alfonso Hernández, Alma Fuentes, Ulrike Keyser, Dolores Padilla, Alba Luz, Ramón Velázquez, Edith Lima, Angélica Bautista y Edith Gutiérrez.

${ }^{14}$ De estas cuestiones la UNAM no está librada. Si bien en el próximo apartado vamos a trabajar con las problemáticas de sexo/género que las/los alumnas/os identificaron en la comunidad universitaria, en este apartado no queremos dejar de mencionar el reciente trabajo de Araceli Mingo y Hortensia Moreno (2015). Las autoras, en un completo y minucioso estudio, el cual va de la teorización al estudio de casos, parten de lo que denominan un "desequilibrio en los posicionamientos simbólicos y prácticos de los sujetos", en donde destacan la violencia contra las mujeres, pero más aún, la "anuencia silenciosa de los integrantes de la organización social donde ocurren", así como "los factores que obstaculizan la denuncia y castigo de quienes perpetran actos de violencia de género, tales como sexismo y hostigamiento sexual" (139). Los ejemplos centrales que conforman su propuesta provienen de experiencias de la UNAM.
} 


\section{Taller crítico y acción comunitaria en el siglo XXI. Perspectivas de género frente a la violencia}

De acuerdo a lo descrito proponemos lo que desde la pedagogía activa se ha denominado "taller crítico" (Andrade y Muñoz, 2004: 253); es decir, una estrategia de trabajo interactivo que permite una aproximación dinámica al conocimiento. Cabe decir cómo esta modalidad de trabajo busca generar diálogos, discusiones, debates y foros, utilizando recursos de seminario, panel, foro, mesa redonda, debate y conversatorio (253). A este respecto, los talleres de género y acción comunitaria aquí presentados parten del seminario teórico para provocar espacios activos de discusión y reflexión, teniéndose, a su vez, el objetivo de realizar "algo", en este caso, la planeación y aplicación de acciones comunitarias concretas, desde una perspectiva "dura" de sexo/género, y adaptadas a la comunidad universitaria de CU en la UNAM. Los talleres, de este modo, funcionaron bajo la siguiente estructura: 1) el comentario y observación de sucesos relacionados con aspectos del orden de sexo/género en México, el mundo y la comunidad universitaria; 2) debates, paneles, mesas redondas y equipos de discusión de los textos teóricos, organizados en los siguientes rubros: feminismo, estudios de género, masculinidades y teoría queer. A estos se suma la lectura de trabajos acerca de la noción "acción comunitaria; y 3) el trabajo en sesiones espaciadas a lo largo del semestre con la planeación, desarrollo, mejora y aplicación de las acciones comunitarias. Durante el primer semestre de cada taller la labor consistió en el proyecto; durante el segundo en su aplicación.

Ahora bien, la noción o teoría de acción comunitaria, si bien ha tenido variantes a largo de la idea moderna de comunidad, ligada a su vez a los procesos educativos no formales (Llena y Úcar, 2006: 1-2), adquiere una remarcada presencia en el siglo XXI. A los cambios en nociones como "local" y "comunidad", o la resignificación de aspectos como la propiedad, la clase social, la estabilidad, el trabajo, la seguridad o la certeza, se suman los conocidos debates en cuanto a los procesos de globalización, la mediación tecnológica y la comunicación mediada, la individualización, la identidad, el riesgo social, la incertidumbre y todas las aceleraciones que hacen de las vidas, en el panorama actual, modos de complejidad (1-2). Para Ricard Gomà (2008) la acción comunitaria resignifica la idea de comunidad, en tanto que al colectivo humano se le reconoce la capacidad de ser sujeto y protagonista de acciones y decisiones. Ahí se explicitan mecanismos y procesos de interacción y apoyo; de pautas de vinculación mutua y reciprocidad cotidiana. Asimismo, el saber/se en un espacio físico, una geografía, que incorpora significados de pertenencia (2008: s.p.). Ahí es donde opera la acción comunitaria. Cabe destacar cómo para una diversidad de autores y enfoques de las acciones comunitarias, su valor parte de la generación de cambios y mejoras sociales, así como del establecimiento, a la vez formal e informal, de formas de trabajo e interacción humana (Gomà, 2008: s.p.). La cuestión final, en todos los casos, es satisfacer necesidades y expectativas de calidad de vida y desarrollo humano, sobre todo mediante relaciones de respeto, confianza y diálogo, fomentándose la transformación y la construcción de ciudadanía (2008: s.p.). A esto podemos agregar una búsqueda de reconstrucción del tejido social, cuestión fundamental en México. Cabe 
destacar cómo otro de los fines que permea todo el proceso es la búsqueda de inclusión, ambiente en el que se entrecruza de lleno con los regímenes de sexo/género y sus lógicas, problemas y violencias. La acción comunitaria, pues, es un tipo de acción social, y si como recuerdan Lombard desde la teorización de género de la violencia, o los autores clásicos, como Connell, Kimmel y las feministas del grueso teórico antes reconstruido, el sexo y el género están en el seno de todo proceso social entonces son un tipo de acción social de sexo/género. Como instancia transformadora, buscan aplicar estrategias y proyectos de acción múltiples (educativos, sociales, residenciales, culturales), buscándose la participación asociativa (Gomà, 2008: s.p.) Igualmente, parten de la capacidad de articular la acción por la igualdad, centrándose en el reconocimiento de las diferencias, de la "diversidad expresada y vivida en positivo" (s.p.).

Volviendo a los talleres, tras dimensionar los temas, aspectos y problemas de sexo/ género principales se establecieron algunos puntos de partida para el planteamiento de las acciones. Así, se determinó trabajar no solamente en los lindes de la FFyL. A este respecto, se consideró que esta facultad representa un lugar privilegiado en términos de información, conocimiento y debates ligados a aspectos de sexo/género. Esto a pesar de que se narraron diversos modos de violencia, destacándose casos de acoso, sobre todo profesor-alumna, aunque también uno de alumno a alumnas (2018-19). Algunos de estos tuvieron bastante repercusión; otros, en cambio, de acuerdo con la lógica bajo la que se siguen rigiendo estos fenómenos en la universidad, no causaron mayor impacto, más que para las víctimas, como lo hemos abordado desde Mingo y Moreno (2015). De acuerdo con esto se consideró la posibilidad de observar y aplicar la metodología de las acciones en otras facultades, en los espacios comunes de la Ciudad Universitaria, e incluso en los $\mathrm{CCH}$, bachilleratos en los que han acontecido una diversidad de eventos violentos en los últimos cursos.

Como se ha expresado, un objetivo común de amplio espectro fue el de hacer llegar los mensajes del feminismo, del género, las masculinidades o la teoría queer a los destinatarios adecuados ${ }^{15}$, por ello es que las acciones comunitarias, como metodologías de reconstrucción del tejido social, en este contexto buscan acabar con mitologías acerca del feminismo y del género; a decir, ideas tales como que estos estudios constituyen espacios "sólo para ellas" -o para los gays y lesbianas-. 0 algunas más graves, pero bien arraigadas desde la cultura popular en la cultura institucional, cifradas en narrativas del tipo: "pero qué exigen si ya tienen todas las libertades e igualdad" o "ellas sólo buscan venganza". Así, un trabajo constante con algunos de estos "lugares comunes" se hizo presente a la hora de plantear los proyectos. La lógica del taller y las distintas dinámicas con las que se discutieron los textos teóricos permitieron apuntar a anécdotas, ejemplos y narrativas circundantes al espacio de la FFyL. P. ej., y de acuerdo con algunos enunciados trabajados en los talleres, podemos nombrar la ya referida feminización de los estudios de género; la construcción de la figura de la feminazi, incluso en espacios académicos y de las IES; la

\footnotetext{
${ }^{15}$ Las problemáticas de violencia de género, así como otros temas ligados al orden de sexo/género, desde una perspectiva académica y profesional consideramos que solamente pueden conocerse, debatirse e interiorizarse si lee sobre feminismo y estudios de género, si se participa en asignaturas y talleres curriculares sobre el tema; o en grupos, asambleas o seminarios.
} 
idea de que las nuevas masculinidades buscan hacer más dócil al hombre para que pueda ser dominado; la de que el feminismo busca el poder de la venganza ${ }^{16}$.

Tras las reflexiones sobre la cotidianeidad del orden de sexo/género se identificaron problemas más específicos. Estos vehicularon las lecturas y debates, y constituyeron los marcos de justificación para el planteamiento de las acciones. Entre estos podemos destacar diversos modos de violencia en las aulas. Si bien los ejemplos de violencia psicológica y física -bullying, acoso verbal, tocamientos, abusos- fueron mucho más llamativos en los casos comentados sobre los CCH y preparatorias, en las aulas de la FFyL se comentaron modos de violencia simbólica y estructural manifestados en "microviolencias", "microinequidades" y "micromachismos". En términos generales, la constante de violencia se abordó en forma verbal o física entre compañeros/as, profesorado, personal dentro y personajes en las inmediaciones; p. ej., en cuanto al comercio formal o informal, destacándose el alto índice de hechos acontecidos en los medios de transporte. Asimismo, formas naturalizadas de violencia por exclusión, en cuanto a pertenencia grupos, realización de actividades, deportes o desempeño en cargos. También se analizaron casos de violencia entre mujeres, dependencia y violencia en el noviazgo, y de extorsión sexual por medios digitales, entre otros.

En cuanto modos de violencia institucional y naturalizada dentro de las lógicas universitarias ligadas a las formaciones y el currículum, se identificaron prácticas y códigos estrictos y restrictivos de sexo/género en facultades de la UNAM como Administración y Contaduría, Derecho e Ingeniería. Estas van desde normativa en el vestido, roles de acuerdo con normas preestablecidas y lugares comunes a la hora de impartirse la educación ${ }^{17}$. Además, espacios en los que se mantienen tradiciones con férreas estructuras de hombres, así como tareas científicas o de pensamiento repartidas según los ideales de sexo/ género $^{18}$; variadas formas de micromachismos, violencia verbal o acoso a mujeres; bajo porcentaje de población gay abierta, etc. De manera alarmante, de acuerdo con varias experiencias de mujeres alumnas de los talleres, presencia de inseguridad, vulnerabilidad, miedo y peligro constante en desplazamientos dentro de CU, sean estos a pie o en transporte público, y más aún hacia en las rutas o medios para regresar al hogar. A este respecto, se identificaron algunos puntos conflictivos y se contrastaron con el "Mapa de riesgo" elaborado por estudiantes de Geografía en el $2018^{19}$. También se trajeron a colación modos de violencia más amplios y naturalizados, como aquellos centrados en inequidad en cuanto a la representación de mujeres, gays, lesbianas y otras identidades sexogenéricas;

\footnotetext{
${ }^{16}$ En esta línea se compartieron pronunciamientos escuchados en espacios de la FFyL, p. ej., la intervención de un alumno que aseveraba que, "si como dicen todas las feministas, los hombres son corruptos, ladrones, traicioneros y han construido un mundo terrible, pues para qué, entonces, quieren convertirse en ellos", entre otros de tal calibre.

${ }^{17}$ En el trabajo referido de Mingo y Moreno (2015) narran el caso de cómo un docente de Ingeniería utiliza una metáfora del funcionamiento de la lavadora para que una alumna pudiera entender un concepto complejo (143). Por otra parte, en la aplicación de algunas de las acciones comunitarias se identificaron testimonios en las que alumnas de Derecho expresaban los rígidos códigos de vestimenta destinados a la mujer en ese ámbito. En uno se podía conocer la opinión de un profesor en cuanto a cómo debían vestir ellas: serias pero sexys.

${ }^{18}$ Kimmel (2017) expresa cómo estos roles se reparten y fijan desde la educación básica. Así, ellas resultan más eficientes a la hora de organizar y sintetizar la información; ellos para el pensamiento y la generación de ideas.

${ }^{19}$ https://www.google.com/maps/d/u/0/viewer?mid=12tCMbkMo0JiXnVt_ok0_3kQjn-LIT_kF\&ll=19.3263455380 $10106 \% 2 \mathrm{C}-99.18292994850071 \& \mathrm{z}=15$.
} 
en cuanto al acceso a puestos de dirección o gestión; participación en actividades escolares, colegiadas, científicas o académicas determinadas por roles y estereotipos; y, finalmente, ignorancia (real o voluntaria) por parte de profesores/as en cuanto a cuestiones, debates, perspectivas y enfoques de feminismo y sexo/género, etc. Ahora, sin poder detenernos en cada uno de los espacios o fenómenos identificados, podemos resumir que en la primera edición -el "Taller de Educación no Formal 3 y 4. Género, estudios culturales y acción comunitaria" (curso 2017-2018)-, en las propuestas de acciones comunitarias se destacaron cuestiones como micromachismos, igualdad y estereotipos de género, feminicidios, e inseguridad y vulnerabilidad. A su vez, en el “Taller de Orientación Educativa I y II. Género, familia y sociedad: taller de acciones comunitarias" (curso 2018-2019), los intereses se concentraron en aspectos como la noción de "techo de cristal", educación de género para la inclusión, violencia en el noviazgo y en el lenguaje cotidiano, o diversidad sexual e inclusión de la diferencia.

En cuanto al carácter teórico-metodológico de las propuestas de acción comunitaria, se decidió en debate en los talleres que cumplieran con los siguientes aspectos:

- Tener como estrategia central una reconstitución del tejido social, bajo una perspectiva "dura" de sexo/género, tomando la FFyL como centro desde el cual observar a la comunidad universitaria, así como los espacios comunes y trayectos.

- Construir una noción de comunidad inclusiva y abiertamente sensible a los regímenes de ordenamiento de sexo/género.

- Buscar horizontalidad, evitándose jerarquías ligadas a la constante de violencia institucionalizada.

- No imponer problemas o soluciones, sino partir de hechos y necesidades claros de la comunidad y las capacidades de todas/os.

- Búsqueda de efectos de mejora, transformación o brindado de seguridad.

- Establecimiento de acciones sociales de sexo/género basadas en la solidaridad, apoyo, intercambio de saberes y herramientas.

\section{Algunas acciones comunitarias}

En el espacio de este trabajo no podemos dar cuenta de todas las acciones comunitarias planteadas y llevadas a cabo, por ello a continuación ofrecemos un breve panorama de las más destacadas.

En el “Taller de Educación no Formal 3 y 4. Género, estudios culturales y acción comunitaria" (curso 2017-2018), Andrea Avilez Ortega, estudiante de Psicología, diseñó la acción “Acompáñame", la cual parte de un objetivo muy claro, basándose en una problemática diaria y de enorme complejidad, que es la de la inseguridad y vulnerabilidad en los traslados hacia y desde la Ciudad Universitaria, o de los movimientos en las rutas internas desde y hacia las facultades. A partir de una noción aparentemente sencilla, la de "acompañarse", la idea parte de una transformación de los trayectos inseguros y violentos en lugares or- 
ganizados, unidos y vinculados para hacer frente a situaciones de molestia, acoso, abuso, violación, e incluso de desaparición forzada. Por ello, esta iniciativa tiene por finalidad el construir redes de acompañamiento de mujeres en la UNAM, o de personas con sentimiento de vulnerabilidad en los traslados. La manera para organizar las rutas de acompañamiento, de acuerdo con trayectos, horarios y necesidades, se ideó de acuerdo a una aplicación para teléfonos inteligentes, que fuera de uso sencillo, de fácil descarga y acceso.

Ahora, esta problemática de partida ha sido un tema de complejidad y debate en tiempos recientes en la Ciudad Universitaria de la UNAM, pues si bien parece haber un número representativo de denuncias y testimonios, así como de casos, la respuesta por parte de las autoridades universitarias no ha sido del todo clara, organizada ni contundente. Según el trabajo de observación de Avilez, la comunidad universitaria vive casi diariamente situaciones de inseguridad y violencia, las cuales aumentan a ciertas horas y en ciertos espacios, siendo las mujeres las más afectadas, pues comúnmente son violentadas, acosadas y hostigadas dentro del espacio universitario. Esta inseguridad se incrementa cuando se desplazan solas. Asimismo, se detectó una insuficiencia de seguridad en la comunidad universitaria, así como falta de protocolos claros en caso de sufrir alguna suerte de acto violento. De este modo, el carácter a la vez dinámico y práctico de esta propuesta surge de dos cuestiones que en ese momento resultaron muy incidentes a nivel internacional, gracias a la eficaz velocidad de la comunicación digital. La primera es la iniciativa “¿Vamos juntas"', de la brasileña Babi Souza, quien a partir de hechos funestos en su país dio pie a un incidente movimiento basado en la aparentemente sencilla premisa: "Cuando te encuentres en una situación de riesgo, observa: al lado tuyo puede estar otra mujer pasando por la misma inseguridad ¿Qué tal si vamos juntas?”20. Asimismo, influyó el movimiento espontáneo que en México surgió a partir del secuestro, violación y asesinato de la estudiante Mara Fernanda Castilla, en Puebla, que ya hemos referido y que dio pie a la campaña "\#MiCasaEsTuCasa”. En términos teóricos, esta acción parte de la lectura de textos esenciales del feminismo o del postestructuralismo, utilizando nociones generales como objetivización o hipersexualización, en la órbita de autoras como Butler o Sayak Valencia, pero aterrizando en la noción de sororidad, establecida por Marcela Lagarde en su incidente trabajo de $2009^{21}$. Finalmente, como estrategia se estableció la identificación de puntos, rutas y horarios de conflicto, además de la realización de encuentros informales entre mujeres y personas en situación de riesgo o vulnerabilidad para compartir testimonios. Además, la politización de las rutas y su función crítica y transformativa.

En otra de las acciones, Luz Elena Rodríguez y Edgar Martínez, ambos estudiantes de pedagogía, diseñaron una campaña de intervención visual en las facultades de Contaduría y Administración, basadas en testimonios obtenidos en estos mismos espacios. El objetivo primordial se planteó bajo la necesidad de informar y sensibilizar en lugares de

\footnotetext{
${ }^{20}$ https://www.movimentovamosjuntas.com.br/.

${ }^{21}$ La autora la define como [...] una dimensión ética, política y práctica del feminismo contemporáneo [...] una experiencia de las mujeres que conduce a la búsqueda de relaciones positivas y a la alianza existencial y política, cuerpo a cuerpo, subjetividad a subjetividad con otras mujeres, para contribuir con acciones específicas a la eliminación social de todas las formas de opresión y al apoyo mutuo para lograr el poderío genérico de todas y al empoderamiento vital de cada mujer" (2006: 126).
} 
la comunidad universitaria en los que los enfoques de género, a la manera de políticas curriculares o estrategias al interior de los programas, no estuvieran del todo presentes; o en los que, según testimonios de los/las alumnos/as se mostraran altos porcentajes de micromachismos sociales y machismo estructural, así también como de violencia y acoso. Tras esto, también se planteó como finalidad el mostrar la relación entre la violencia cotidiana y el asesinato por cuestiones de sexo/género.

Como planteamiento a partir de la teoría, el trabajo que influyó más los preceptos de lectura fue el celebrado Todos deberíamos ser feministas (2014) de Chimamanda Ngozi Adichie. Como se ha expresado, en su reconstrucción de la vida familiar y social de su natal Nigeria desde una perspectiva de sexo/género, resulta revelador lo parecidas que resultan las sociedades nigeriana y mexicana. Además, el perspicaz ojo con el que esta autora identifica los espacios mínimos de la reproducción de la opresión y diferencia sexuales resultan de especial ayuda a la hora de observar lugares como los de la educación superior. En el marco teórico también se refirieron nociones de roles y estereotipos, equidad y la idea en sí de "diferencia sexual” en Rubin y Butler, además de la definición de patriarcado (Friedan y Firestone), o la de política sexual de Millet. La estrategia consistió en la aplicación de un pretest a grupos representativos de dichas facultades, centrados en el conocimiento y reflexión de aspectos ligados a la violencia de género. Después, en el diseño y pegado de carteles informativos en las zonas de más afluencia de dichas facultades, con carácter de comunicación de impacto y reflexión, basados en los testimonios obtenidos. Después, la aplicación de un postest para analizar cambios en la percepción de la violencia cotidiana, así como de los micromachismos y el machismo estructural. Entre los resultados podemos destacar un alto porcentaje de apreciación de estos fenómenos tras la campaña de intervención visual. Además, la identificación de por lo menos tres casos confesados de acoso por parte de profesores a alumnas bajo la forma de insinuaciones verbales, invitaciones a cenas o salidas y tocamientos. También se identificaron la exclusión de grupos, eventos o actividades por cuestiones de sexo/género, o la abierta y cínica negación de los sujetos varones a reconocer el machismo, la violencia o los feminicidios como tales ${ }^{22}$.

En la versión del taller del curso 2018-2019 queremos destacar el giro hacia la comunicación y gestión digital que dieron las acciones. A este respecto, se dio un entrecruce interesante entre género y las llamadas pedagogías digitales. Sin duda, las/los alumnas/ os de estas generaciones conciben el universo de las redes sociales como un espacio natural para el establecimiento de modos de educación no formal, de implementación de acciones sociales, y de modos de activismo, resistencia y transformación. De hecho, con el conocimiento de redes digitales de las/los miembros a lo largo del curso se hizo un seguimiento muy cercano a los claros enfoques de género presentes en estas, así como a las iniciativas y debates en esta línea.

${ }^{22}$ En el marco de esta primera edición del taller también se trabajaron los micromachismos en una acción pedagógica denominada "El micro-reflejo", llevada a cabo con un grupo de 40 estudiantes de la Escuela Nacional Preparatoria No. 8. Esta fue diseñada por las estudiantes de pedagogía María Fernanda Gutiérrez, Sofía Mendoza y Mariana Perujo, incluyendo con dinamismo nociones no sólo de feminismo, sino de la teoría de las masculinidades. En este contexto resultó alarmante el tema del acoso por parte de compañeros mayores, profesorado y adultos de la comunidad estudiantil, alrededores y la familia en general, así como lo normalizado que esta realidad se presenta. 
Entre las acciones comunitarias de este periodo destacamos "El amor no mata", de Emily Chavez Rosas y Janneth Irán Ríos, estudiantes de Pedagogía. En esta se parte de la sencilla pregunta: “A qué están dispuestas las mujeres por amor?”, reparándose en cómo la violencia al interior de la pareja, especialmente en jóvenes estudiantes de universidad, posee una notable presencia y repite patrones en perfiles muy variados. Tras una medición de los posibles niveles de violencia al interior del noviazgo -desde la prohibición de usar determinada prenda hasta los golpes- esta propuesta surge de una interesante reflexión acerca de cómo se vive esta realidad en la FFyL de la UNAM, en los tiempos que corren y teniéndose como telón de fondo la comunicación digital. En una facultad con el 78.4\% de mujeres la propuesta aboga por una toma de conciencia, apelando directamente a la población femenina en cuanto a cómo se viven las relaciones, y dentro de estas, una diversidad de expresiones de control, dominio y otras formas de la constante de violencia sexual y de género. Cabe destacarse cómo el trabajo parte también de hechos puntuales, como la violación de una alumna, en el 2018, en el estacionamiento de la Facultad, o el referido feminicidio de Lesvy Berlín. A partir de estos se reconstruye una suerte de escenario en el que la violencia, si bien tiene grados, está interrelacionada. El objetivo de la acción se planteó, así, como el de la configuración de un espacio digital -un blog y una página de Facebook $^{23}$ - como lugar de diálogo, compartición de experiencias y de apoyo de "mujeres para mujeres", detectándose con esto modos de violencia en el noviazgo y situaciones en las que la integridad o vida de alumnas pudiera correr peligro. En el planteamiento se establecieron pertinentes referencias al conocido "Violentómetro", herramienta impulsada por alumnos y académicos del Instituto Politécnico Nacional (2009) ${ }^{24}$. Esta acción también contó con una campaña visual en la FFyL, lo cual logró un interés considerable, y su funcionamiento ligó el blog, en el cual las usuarias encontraron información pertinente acerca de una diversidad de temas sobre violencia en la pareja, con la página de Facebook, en donde las impulsoras recibieron de manera privada, anónima y confidencial testimonios de alumnas. Ejerciendo como administradoras y facilitadoras de la comunicación su labor consistió en adaptar los relatos o ideas compartidos para su publicación. En dos casos de gravedad conectaron a las usuarias con una psicóloga profesional en temas de violencia de género. Entre los textos teóricos de los que partió esta propuesta se encuentra la bibliografía general del curso, además de bibliografía extra y específica sobre el tema. Desde esta se fue informando a las usuarias de los soportes digitales, y se ahondo en términos recientes, como el de gaslighting, que sería una estrategia mediante la cual una persona es constantemente bombardeada con aseveraciones que menguan su identidad, seguridad, autopercepción o saludo, p. ej: "te enojas por todo", “estás loca”, "la culpa fue tuya", "te imaginas cosas", "eres una celosa", etc.

Otra acción destacada es la planteada por Brenda Margarita Güevara Cárdenas, estudiante de Historia, y Luis Sergio Muthe Paez, de Pedagogía. Bajo el nombre "Palabras sin violencia" esta propuesta se centra en la violencia sexual o de género de carácter verbal.

\footnotetext{
${ }^{23}$ Nombre de la página: "El amor no mata - FFyL".

${ }^{24}$ https://www.ipn.mx/genero/materialesdeapoyo/violentometro.html.
} 
En su planteamiento acometen una honda reflexión en cuanto al papel performático/performativo del lenguaje, ligándolo a la violencia cotidiana, naturalizada o consensuada con carácter discriminador y dominante, en términos machistas, misóginos, homofóbicos y de violencia en general, pues como se ha venido argumentando, el orden de sexo/género puede situarse en la base de toda violencia. Con una notable sensibilidad a la comunicación digital, los impulsores de esta acción argumentaron con ejemplos de gran actualidad, como en el caso de frases virales cuyo contenido lúdico borra el carácter violento de violencia de género, p. ej. en la frase "cállese viejo lesbiano", que fue "trending topic" durante días en México y otras redes de habla hispana. Asimismo, también se llevó a cabo un análisis más tradicional en el que, desde una perspectiva educativa, se analizó cómo se construyen las identidades, de manera negativa, desde frases cotidianas como "no seas puto" o "lloras como niña". Finalmente, basándose en las lecturas sobre teoría de las masculinidades, en cuanto a la noción de Connell de masculinidad hegemónica, así como en un marco más amplio de teoría feminista, como con las descripciones de Betty Friedan en su Mística de la feminidad, el resultado final fue un dinámico sitio web en el que los usuarios podían realizar diversos tests para conocer su grado de naturalización de violencia verbal de género, o para entrar en contacto con material sobre el tema.

\section{Conclusiones y vista a futuro}

Cerramos este trabajo remarcando algunas de las cuestiones principales que se desprenden del recorrido planteado: de teoría a la acción. En primer lugar, como hemos intentado mostrar, la teoría feminista y de género, incluyendo a las masculinidades y los estudios queer, son algo más que la representación de un mundo injusto, desequilibrado o basado en lógicas de poder o dominación, ya que superan los lindes de un modo de explicación de los fenómenos del mundo o un punto de vista de la realidad. Son, en conjunto, un material para transformar, incidir y rehacer el tejido social. Esta especial fuerza es en el espacio del campo universitario en donde puede -y debe- alcanzar una articulación central. Esto, pensamos, no sólo porque otorga las herramientas para que las identidades de los alumnos se construyan e inserten en el contexto de un mundo cambiante, acelerado y global -según las conocidas narrativas del presente-, sino porque posee los mensajes y las armas epistemológicas, políticas y culturales para hacer de las vidas experiencias mejores. Atacar el principio de diferencia sexual desde una diversidad de frentes es luchar contra la violencia instaurada, como régimen de sexo/género, en el ordenamiento mayor de la sociedad.

En segundo término, la posibilidad de que la constante de violencia sea concebida como violencia de sexo/género en sí permite articular los modos en los que esta se ataja desde instancias a la vez novedosas e incidentes. Como pudimos observar, desde una diversidad de trabajos se ha planteado que la diferencia sexual -y toda la serie de nociones y problemas que de esta irradian- se encuentra en la base de todas las relaciones -educativas, sociales, culturales, políticas-. Asimismo, si la violencia, como el problema mayúsculo de ese haz de relaciones, se puede situar, igualmente, en el centro de estas, con esto esta- 
ríamos comprobando cómo es que la dimensión violenta de la experiencia humana ha de tratarse como un tema de feminismo y de género. Además, en la breve reconstrucción que hemos presentado sobre violencia y género hemos mostrado cómo las diversas autoras y autores (Connell, Kimmel, Lombard, Mingo) derivan sus lecturas, análisis y teorizaciones hacia el espacio de la masculinidad. Por ello, en un sistema de relaciones en donde los varones mantienen la posición dominante; sistema que se perpetúa y reproduce en todas las esferas de la experiencia, en el que se mantienen ciertos privilegios, y las identidades no hegemónicas son vistas como débiles, secundarias o complementarias en el mejor de los casos, el continuo de la violencia se presenta como la instancia principal para su mantenimiento, mostrándose la cara de cierta noción de masculinidad y sus prácticas.

Ahora bien, si la violencia es fundamentalmente masculina, su combate, en espacios de enorme influencia y representatividad, como el de las aulas de la universidad y sus espacios comunes, ha de provenir de perspectivas, políticas, didácticas y currículos con enfoques "duros" de sexo/género, pero también globales; es decir, basados en las premisas del feminismo aunque en dialogo nutrido con los estudios de la masculinidad o la teoría queer, y sus propuestas de otros modos de vivir la masculinidad o de deconstruirla. Finalmente, insistimos en la oportunidad que otorgan formas destacadas de educación no formal, y que bien pueden acometer un acto educativo en "sentido contrario"; es decir, ir de los espacios de lo cotidiano a la institución, situándose como tecnologías de transformación "entre mundos". Su fin más alto, no lo olvidemos, es el de la reconstrucción del tejido social y esta acción sólo podrá ser si se hace bajo la atenta visión del sistema sexo/género.

\section{Bibliografía}

ANDRADE, MARTHA y MUÑOZ, CLARENA. (2004). "El taller crítico: Una propuesta de trabajo interactivo". Tabula Rasa. Revista de Humanidades, (no. 2), pp.251-262.

BUTLER, JUDITH. (1990 [2007]). El género en disputa. Barcelona: Paidós.

ADICHIE, CHIMAMANDA N. (2015 [2011]). Todos deberíamos ser feministas. Barcelona: Random House.

CONNELL, W. RAEWYN. (1995). Masculinities. Berkeley: University of California Press.

DELGADO, GABRIELA. (2008). "Metodología de la investigación con perspectiva de género". En Vázquez, María de Lourdes y Mireles, Olivia (Coords.). Metodología de la investigación. La visión de los pares (17-38). México: IISUE-UNAM.

GOMÁ, RICARD. (2008). "La acción comunitaria: transformación social y construcción de ciudadanía". Revista de Educación Social, (núm 7) 3-7.

GONZÁLEZ, ROSA ET AL. (2012), “Cómo entendemos la violencia de género en las instituciones de educación superior?", en Géneros. Revista de investigación y divulgación sobre los estudios de género, no. 11, época 2, pp. 80-96.

FIRESTONE, SHULAMITH. (1976 [1970]). La dialéctica del sexo. Barcelona: Kairós. FRIEDAN, BETTY. (2016 [1963]). La mística de la feminidad. Madrid: Ediciones Cátedra. KIMMEL, MICHAEL. (2017 [2000]). The Gendered Society. Nueva York: Oxford UP. 
KOSOTSKY, EVE. (2002 [1993]). “A(queer) y ahora”. En Mérida, Rafael. Sexualidades transgresoras: una antología de estudios queer (29-55). Barcelona: Icaria.

LAGARDE, MARCELA. (2006). "Pacto entre mujeres: sororidad". Revista Aportes, (vol. 25),123-135.

LLENA, ASUN y ÚCAR, XAVIER. (2006). “Acción comunitaria: miradas y diálogos interdisciplinarios". En Llena, Asun y Úcar, Xavier. (coords). Miradas y diálogos interdisciplinares e interprofesionales. Barcelona: Graó.

LOMBARD, NANCY. (2018). "Introduction to gender and violence". En Lombard, Nancy. The Routledge Handbook of Gender and Violence (1-13). New York: Routledge.

MILLET, KATE. (1969). Política sexual. Madrid: Anaya.

MINGO, ARACELI. (2010). "Ojos que no ven... Violencia escolar y género". Revista Perfiles Educativos, vol. XXXII (núm 130), 25-48.

MINGO, ARACELI y MORENO, HORTENCIA. (2015). "El ocioso intento de tapar el sol con un dedo: violencia de género en la universidad". Revista Perfiles Educativos, vol. XXXVII (núm 148), 138-155.

PRECIADO, BEATRIZ. (2002). Manifiesto contrasexual. Barcelona: Anagrama.

RUBIN, GAYLE. (2000 [1996]). "El tráfico de mujeres: Notas sobre la 'economía política' del sexo". En Lamas, Marta (comp.). El género. La construcción cultural de la diferencia sexual (35-96). México: Porrúa.

ZAMUDIO-SÁNCHEZ et al. (2017), "Violencia de género sobre estudiantes universitarios/as”, en Convergencia. Revista de Ciencias Sociales, UAEM, no. 75, pp. 133-157. 